\title{
Sequelae of Nonsustained Polymorphic Ventricular Tachycardia Induced During Programmed Ventricular Stimulation
}

\author{
William H. Kou, MD, Michael de Buitleir, MD, Alan H. Kadish, MD, and Fred Morady, MD
}

\begin{abstract}
The results of 206 programmed ventricular stimulation studies performed in 130 patients (100 men and 30 women, mean age $62 \pm 12$ years, \pm standard deviation) were examined prospectively to determine the sequelae of nonsustained polymorphic ventricular tachycardia (VT) induced during programmed ventricular stimulation. The clinical indication for the electrophysiologic study was either documented monomorphic VT or unexplained syncope. The pacing protocol included 2 right ventricular pacing sites, 2 basic drive cycle lengths and up to 3 extrastimull. In 111 studies, nonsustained polymorphic VT was induced and with continuation of the programmed stimulation protocol, sustained monomorphic VT was induced in 48 studies $(43 \%)$ and polymorphic VT was induced in $\mathbf{1 3}$ studies
\end{abstract} (12\%). Overall, sustained monomorphic VT was induced in 110 studles and sustained polymorphic $V T$ in 18 studies. The incidence of nonsustained polymorphic VT preceding the induction of sustained polymorphic VT was significantly greater than the incidence of nonsustained polymorphic VT preceding the induction of sustained monomorphic VT (72 vs 44\%, p <0.05). Nonsustained polymorphic VT is not a useful predictor of the outcome of programmed ventricular stimulation. The use of nonsustained polymorphic VT as an endpoint for stimulation would be likely to improve the specificity of programmed ventricular stimulation by limiting the induction of sustained nonclinical arrhythmias that require countershock, but at the cost of significanthy impairing the yield of monomorphic vT.

(Am J Cardiol 1989,64:1148-1151)
From the Division of Cardiology, Department of Internal Medicine, University of Michigan Medical Center, and the Section of Cardiology, Ann Arbor Veterans Administration Medical Center, Ann Arbor, Michigan. Manuscript received February 24, 1989; revised manuscript received July 3, 1989, and accepted July 27 .

Address for reprints: William H. Kou, MD, Section of Cardiology (111A), Ann Arbor Veterans Administration Medical Center, 2215 Fuller Road, Ann Arbor, Michigan 48105.
Tn n patients who do not have a history of ventricular fibrillation or cardiac arrest, polymorphic ventricular tachycardia (VT) or ventricular fibrillation induced by programmed ventricular stimulation may often be laboratory artifacts that do not have clinical significance. ${ }^{1-3}$ Direct current countershock is needed to terminate these arrhythmias if they are sustained, and therefore it is desirable to avoid their induction. Limiting the number of extrastimuli to 2 would significantly reduce the incidence of nonclinical arrhythmias during programmed ventricular stimulation; however, this would have the undesirable effect of also reducing the yield of clinically important VT. ${ }^{4-6}$

We have observed that the induction of sustained polymorphic VT and ventricular fibrillation during programmed ventricular stimulation is often preceded by the induction of nonsustained polymorphic VT. Therefore, it might be possible to avoid the induction of sustained polymorphic VT or ventricular fibrillation by moving to the next step in the stimulation protocol once nonsustained polymorphic VT is induced. However, it is unclear whether this practice would also limit the induction of clinical forms of VT. Therefore, the present prospective study was designed to determine the eventual outcome of programmed ventricular stimulation once nonsustained polymorphic VT is induced, and how often the induction of clinical VT and sustained nonclinical forms of VT/ventricular fibrillation is preceded by the induction of nonsustained polymorphic VT.

\section{METHODS}

Characteristics of subjects: Table I lists the clinical characteristics of the subjects in this prospective study. There were 130 patients ( 100 men and 30 women) with a mean age of $62 \pm 12$ years ( \pm standard deviation). These patients underwent electrophysiologic testing because of a history of either documented monomorphic VT or unexplained syncope. Because of the uncertain significance of sustained polymorphic VT and ventricular fibrillation initiated by programmed ventricular stimulation in patients without a history of cardiac arrest or spontaneous ventricular fibrillation, patients undergoing an electrophysiologic study for either of these 2 indications were not included in the present study. In 76 patients, the left ventricular ejection fraction was determined either by multigated radionuclide or contrast ventriculography.

Antiarmythmic therapy: One hundred fourteen studies were performed in the control state after all antiarrhythmics had been withheld for at least 5 half-lives. The remaining 92 studies were performed while patients 
were being treated with antiarrhythmic medications, after induction of sustained monomorphic VT in a control study. Drug studies with type I antiarrhythmic medications were performed after the patient had received $\geq 5$ doses of the agent. Drug studies with amiodarone were performed after a loading dose of $1,200 \mathrm{mg} /$ day for 2 weeks.

Programmed ventricular stimulation study: Electrophysiologic studies were performed in the postabsorptive, nonsedated state after informed consent was obtained. Two 6Fr quadripolar electrode catheters were inserted percutaneously into a femoral vein and positioned against the right ventricular apex and outflow tract or septum under fluoroscopic guidance. Leads $V_{1}$, I and III and the intracardiac electrocardiograms were recorded on a Siemens Elema Mingograf 7 recorder at a paper speed of $25 \mathrm{~mm} / \mathrm{s}$. If monomorphic VT was induced, a 12-lead electrocardiogram was recorded whenever possible. Stimulation was performed with a programmable stimulator (Bloom Associates) using stimuli that were twice diastolic threshold and $2 \mathrm{~ms}$ in duration.

The details of the stimulation protocol have been described previously. ${ }^{7}$ In brief, 1 and 2 extrastimuli were delivered at the right ventricular apex and outflow tract or septum at basic drive cycle lengths of 600 and 400 $\mathrm{ms}$. If sustained VT was not induced, a third extrastimulus was added and programmed stimulation was repeated at both sites. The coupling intervals of the extrastimuli were limited to $180 \mathrm{~ms}$ because a previous study demonstrated that restriction of the coupling intervals of the extrastimuli to $180 \mathrm{~ms}$ reduces the incidence of nonclinical forms of VT without compromising the yield of clinically significant VT. ${ }^{7}$

The endpoint of the stimulation protocol was either the induction of sustained VT or ventricular fibrillation or completion of the pacing protocol. Sustained VT was defined as VT that lasted $>30$ seconds in duration or required termination because of hemodynamic compromise. Nonsustained VT was defined as VT that lasted 3 beats to 30 seconds. Polymorphic VT was defined as VT that showed a beat-to-beat variation in QRS morphology. When multiple episodes of nonsustained polymorphic VT were induced at a particular step of the stimulation protocol, only the longest episode of nonsustained VT was noted for analysis.

Analysis of data: Data are expressed as mean \pm standard deviation. The statistical analyses were performed using either a chi-square analysis or a Student $t$ test. A p value $<0.05$ was considered statistically significant.

\section{RESULTS}

Sequelae of induced nonsustained polymorphic ventricular tachycardia: Nonsustained polymorphic VT was induced in 111 of 206 studies (54\%). With continuation of the pacing protocol, the sequelae of these 111 studies consisted of induction of sustained monomorphic VT in 48 studies (43\%), sustained polymorphic VT in 13 studies $(12 \%)$ and no sustained VT in the remaining 50 studies $(45 \%)$.

\begin{tabular}{|lc|}
\hline TABLE I Patient Characteristics \\
\hline Sex: M/F & $100 / 30$ \\
Age (yrs, mean I standard deviation) & $62 \pm 12$ \\
Underlying heart disease & \\
Coronary artery disease & 88 \\
Valvular heart disease & 10 \\
Dilated cardionyopathy & 7 \\
Systemic hypertension & 5 \\
None & 20 \\
Indication for electrophysiologic study & \\
Documented monomorphic ventricular tachycardia & 88 \\
Unexplained syncope & 42 \\
\hline
\end{tabular}

TABLE II Number of Studies in Which 1, 2 and 3 Extrastimuli Were Used to Induce Sustained Ventricular Tachycardia and Nonsustained Polymorphic Ventricular Tachycardia

\begin{tabular}{|llll|}
\begin{tabular}{llll|} 
No. & Sustained \\
of & Monomorphic & Sustained & Polymorphic \\
VT
\end{tabular} & $\begin{array}{l}\text { Nonsustained } \\
\text { Polymorphic } \\
\text { VT }\end{array}$ \\
\hline 1 & VT & 0 & 9 \\
2 & 26 & 3 & 38 \\
3 & 55 & 15 & 64 \\
\hline VT = ventricular tachycardia. & & \\
\hline
\end{tabular}

In 95 studies, nonsustained polymorphic VT was not induced. The outcome of these studies was sustained monomorphic VT in 62 studies (65\%), sustained polymorphic VT in 5 studies $(6 \%)$ and no sustained VT in the remaining 28 studies ( $29 \%$ ). The incidence of these outcomes was not significantly different than in studies in which nonsustained polymorphic VT was induced.

Incidence of nonsustained polymorphic ventricular tachycardia induced before the induction of sustained ventricular tachycardia: Sustained monomorphic VT was induced in 110 of 206 studies, and 48 of 110 (44\%) were preceded by the induction of nonsustained polymorphic VT. Sustained polymorphic VT was induced in 18 of 206 studies, and 13 of $18(72 \%)$ were preceded by the induction of nonsustained polymorphic VT. The incidence of nonsustained polymorphic VT preceding the induction of sustained polymorphic VT was significantly greater than the incidence preceding the induction of sustained monomorphic VT $(p<0.05)$.

The number of studies in which single, double and triple extrastimuli were used to induce sustained and nonsustained VT is listed in Table II.

Duration and cycle length of Induced nonsustalned polymorphic ventricular tachycardia before the induction of sustained ventricular tachycardia: There was no correlation between the duration of the induced nonsustained polymorphic VT and the type of sustained VT subsequently induced. The maximal duration of nonsustained polymorphic VT preceding the induction of sustained monomorphic VT was $8 \pm 9$ beats, compared to $14 \pm 13$ beats when preceding the induction of sustained polymorphic VT (difference not significant).

The mean cycle length of the induced nonsustained polymorphic VT preceding the induction of sustained polymorphic VT was shorter than that preceding the induction of sustained monomorphic VT $(235 \pm 46$ vs $313 \pm 70 \mathrm{~ms}, \mathrm{p}<0.05)$. 
POLYMORPHIC VENTRICULAR TACHYCARDIA

\begin{tabular}{|c|c|c|c|}
\hline & $\begin{array}{l}\text { Sustained } \\
\text { Polymorphic } \\
\text { VT }\end{array}$ & $\begin{array}{l}\text { Sustained } \\
\text { Monomorphic } \\
\text { VT }\end{array}$ & $\begin{array}{l}\text { Nonsustained } \\
\text { Polymorphic } \\
\text { VT }\end{array}$ \\
\hline Control (\%) & $13(11)$ & $38(33)$ & $70(61)$ \\
\hline \multirow[t]{2}{*}{ Drug follow-up (\%) } & $5(5)$ & $72(78)$ & $41(45)$ \\
\hline & NS & $p<0.001$ & $p<0.025$ \\
\hline
\end{tabular}

Induction of nonsustained monomorphic ventricular tachycardia before the induction of sustained monomorphic ventricular tachycardia: In 58 of 206 studies, nonsustained monomorphic VT was induced during programmed ventricular stimulation. Thirty-nine (67\%) were followed by the induction of sustained monomorphic VT and only $10(17 \%)$ of these 39 nonsustained VTs had a morphology and cycle length similar to the sustained VT subsequently induced. The mean duration of these nonsustained monomorphic VTs was $16 \pm 17$ beats (range 3 to 58 beats).

Cycle length of sustained monomorphic ventricular tachycardia and the induction of nonsustained polymorphic ventricular tachycardias The mean cycle length of the 48 sustained monomorphic VTs that were preceded by the induction of nonsustained episodes of polymorphic VT was $303 \pm 73 \mathrm{~ms}$ (range 220 to 490 $\mathrm{ms}$ ). The mean cycle length was $358 \pm 84 \mathrm{~ms}$ (range 220 to $595 \mathrm{~ms}$ ) for the 62 monomorphic VTs whose induction was not preceded by nonsustained polymorphic VT (difference not significant).

Left ventricular function and the induction of sustained polymorphic ventricular tachycardia: The left ventricular ejection fraction was measured in 76 patients who had a total of 127 studies. In patients who had a left ventricular ejection fraction $>40 \%$, sustained polymorphic VT was induced in 1 of 33 studies (3\%). In patients who had a left ventricular ejection fraction $<40 \%$, sustained polymorphic VT was induced in 6 of 94 studies $(6 \%)$. There was no significant difference in the incidence of sustained polymorphic VT between these 2 groups of patients.

Comparison of control and drug studies: A comparison of the control and drug follow-up studies demonstrated no significant differences in the incidence of sustained polymorphic VT (11\% of drug follow-up vs $5 \%$ of control, difference not significant) during programmed ventricular stimulation (Table III). There was a higher incidence of sustained monomorphic VT (78 vs $33 \%$ of control, $\mathrm{p}<0.001$ ) and a lower incidence of nonsustained polymorphic VT ( 45 vs $61 \%$ of control, p <0.05) in drug follow-up studies.

\section{DISCUSSION}

Predictive value of nonsustained polymorphic ventricular tachycardia during programmed ventricular stimulation: The results of this study demonstrate that a majority (72\%) of nonclinical sustained polymorphic VTs that are induced by programmed ventricular stimulation and that require countershock to terminate are preceded by the induction of nonsustained polymorphic VT. It is therefore possible that the induction of a large percentage of sustained nonclinical VTs could be avoided if stimulation at a particular step of the stimulation protocol was discontinued on induction of nonsustained polymorphic VT. However, $>40 \%$ of clinically important monomorphic VTs were also preceded by the induction of nonsustained polymorphic VT. Therefore, the use of nonsustained polymorphic VT as an endpoint for stimulation at a particular step of a programmed stimulation protocol would be likely to improve the specificity of programmed ventricular stimulation, but at the cost of significantly impairing the yield of monomorphic VT.

The mean duration of the nonsustained polymorphic VT that preceded the induction of sustained polymorphic VT and sustained monomorphic VT did not differ significantly. Although the mean cycle length of induced nonsustained polymorphic VT was shorter in studies in which sustained polymorphic VT was subsequently induced than in those in which sustained monomorphic VT was subsequently induced, there was much overlap in the range of cycle lengths and a critical cycle length, below which only the induction of nonclinical VT/ventricular fibrillation was likely to occur, could not be indentified. Therefore, there are no characteristics of nonsustained polymorphic VT that are helpful in improving its value as a predictor of sustained polymorphic VT. In addition, neither the cycle length of the patient's clinical VT nor the left ventricular ejection fraction influences the predictive value of nonsustained polymorphic VT.

Configuration of nonsustained monomorphic ventricular tachycardia: In this study, $<20 \%$ of induced nonsustained monomorphic VTs were similar to configuration to the sustained monomorphic VTs later induced. Therefore, nonsustained monomorphic VT induced during programmed stimulation is not an accurate predictor of the type of sustained monomorphic VT that may be subsequently induced.

Subjects studied: Our subjects were limited to patients who had either a documented history of unimorphic VT or unexplained syncope. Because none of the patients studied had a history of sustained polymorphic VT requiring countershock, it could be concluded that sustained polymorphic VT induced by programmed ventricular stimulation in these patients was most likely a "nonclinical" arrhythmia. Furthermore, in patients with unexplained syncope, the results of previous studies have demonstrated that monomorphic VT induced by programmed ventricular stimulation is a clinically important arrhythmia, whereas polymorphic VT induced during programmed ventricular stimulation does not have clinical significance. 8,9 Therefore, the inclusion of only patients who had a documented history of monomorphic VT or unexplained syncope allowed us to reliably classify induced arrhythmias as clinical or nonclinical. However, the possibility that polymorphic VT was clinically important in some patients cannot be ruled out. 
The results of programmed ventricular stimulation are highly dependent on the population of patients undergoing electrophysiologic testing. It should therefore be noted that the sequelae of nonsustained polymorphic VT reported in the present study might not be applicable in groups of patients that differ in composition from the subjects of the present study. For example, the yield of sustained monomorphic VT would be expected to be lower in patients who did not have a history of VT than in patients with a documented history of VT.

Limitations: Forty-five percent of the electrophysiologic studies were performed in the presence of antiarrhythmic therapy. These cases represent a subgroup of patients who were more likely to have inducible monomorphic VT when undergoing repeat electrophysiologic testing. A potential bias may have been introduced into the results, as data from $>1$ study were collected in these patients.

Clinical implications: During programmed ventricular stimulation, it is desirable to avoid the induction of sustained nonclinical forms of polymorphic VT that require cardioversion. Although the induction of nonsustained polymorphic VT may be a harbinger of sustained polymorphic VT if stimulation is continued at the same step of the stimulation protocol, the results of this study indicate that discontinuation of stimulation may significantly diminish the yield of clinical forms of monomorphic VT, at least in patients who are undergoing electrophysiologic testing because of a history of monomorphic VT or unexplained syncope. Therefore, programmed stimulation should be continued if nonsus- tained polymorphic VT is induced. If the coupling intervals are limited to $180 \mathrm{~ms}$, sustained monomorphic VT will be a sequelae of nonsustained polymorphic VT much more commonly than will be sustained polymorphic VT.

Acknowledgment: We extend our appreciation to Penny Weaver for excellent secretarial assistance.

\section{REFERENCES}

1. Morady F, Shapiro W, Shen E, Sung RJ, Scheinman MM. Programmed ventricular stimulation in patients without spontaneous ventricular tachycardia. Am Heart J 1984; J07:875-882.

2. DiCarlo LA, Morady F, Schwartz AB, Shen EN, Baerman JM, Krol RB, Scheinman MM, Sung RJ. Clinical significance of ventricular fibrillation-flutter induced by ventricular programmed stimulation. Am Heart J 1985;109:959-963. 3. Brugada P, Green M. Abdollah H, Wellens HJJ. Significance of ventricular arrhythmias initiated by programmed ventricular stimulation: the importance of the type of ventricular arrhythmias induced and the number of premature stimuli required. Circulation 1984,69:87-92.

4. Buxton AE, Waxman HL, Marchlinski FE, Untereker WJ, Waspe LE, Josephson ME. Role of triple extrastimuli during electrophysiologic study of patients with documented sustained ventricular tachyarrhythmias. Circulation $1984 ; 69: 532-540$.

5. Platia EV, Greene HL, Vlay SC, Werner JA, Gross B, Reid PR. Sensitivity of various extrastimulus techniques in patients with serious ventricular arrhythmias. Am Heart J 1983;106:698-703.

6. Prystowsky EN, Miles WM. Evans JJ. Induction of ventricular tachycardia during programmed electrical stimulation: analysis of pacing methods. Circulation 1986:73(suppl II):32-38.

7. Morady F, DiCarlo LA, Baerman JM, de Buitleir M. Comparison of coupling intervals that induced clinical and nonclinical forms of ventricular tachycardia during programmed stimulation. Am J Cardiol 1986;57:1269-1273.

8. Morady F, Shen E, Schwartz A, Hess D, Bhandari A, Sung RJ, Scheinman MM. Long-term follow-up of patients with recurrent unexplained syncope evaluated by electrophysiologic test-up. JACC 1983;2:1083-J089.

9. Akhtar M, Shenasa M, Denker S, Gilbert C, Rizwi N. Role of cardiac electrophysiologic studics in patients with unexplained recurrent syncope. $P A C E$ 1983:6:192-201. 